\title{
Paper Pilot Design for Sparse Channel Estimation in Orthogonal Frequency Division Multiplexing Systems
}

\author{
P. Vimala and G. Yamuna \\ Annamalai University, Annamalai Nagar, Chidambaram, Tamil Nadu, India
}

https://doi.org/10.26636/jtit.2018.113817

\begin{abstract}
Orthogonal Frequency Division Multiplexing (OFDM) is a well-known technique used in modern wide band wireless communication systems. Coherent OFDM systems achieve its advantages over a multipath fading channel, if channel impulse response is estimated precisely at the receiver. Pilot-aided channel estimation in wide band OFDM systems adopts the recently explored compressive sensing technique to decrease the transmission overhead of pilot subcarriers, since it exploits the inherent sparsity of the wireless fading channel. The accuracy of compressive sensing techniques in sparse channel estimation is based on the location of pilots among OFDM subcarriers. A sufficient condition for the optimal pilot selection from Sylow subgroups is derived. A Sylow subgroup does not exist for most practical OFDM systems. Therefore, a deterministic pilot search algorithm is described to select pilot locations based on minimizing coherence, along with minimum variance. Simulation results reveal the effectiveness of the proposed algorithm in terms of bit error rate, compared to the existing solutions.
\end{abstract}

Keywords-channel estimation, compressive sensing, minimum coherence, minimum variance, pilot pattern.

\section{Introduction}

Orthogonal Frequency Division Multiplexing (OFDM) is a multi-carrier modulation technique used in wideband wireless communication systems due to its high spectral efficiency [1]. In OFDM systems, each subcarrier has a narrow bandwidth which ensures signal robustness against frequency selectivity caused by the multipath delay spread. Although coherent, non-coherent and partially coherent detection techniques are deployed in OFDM systems, coherent detection attracts wider interest, as it supports a higher date rate than the other schemes. Coherent communication in OFDM systems allows arbitrary signal constellations for high data rates, but efficient channel estimation strategies are required for detection and decoding of information at the receiver. The channel can be estimated in the receiver using the pilot-aided method, where the receiver makes use of the known transmitted symbols, also known as pilots in, a set of predetermined subcarriers of the OFDM system, or by learning the statistics of the information bearing signals which are referred to as a blind technique. As such an approach involves more complex signal processing and computationally expensive operations to ensure efficient blind methods, pilot-aided methods evoke considerable interest in the recent technologies which are capable of tracking the channel based on coherence time whose duration is in the order of one OFDM symbol [2].

The majority of research concerned with pilot-aided channel estimation is devoted to the selection of pilot locations. Equally spaced pilot locations become the optimal selection when the maximum likelihood (ML) rule is employed in the receiver, which is computationally expensive [3], [4]. Further, several investigations show that many wideband OFDM systems tend, in practice, to have their wireless channel dominated by a relatively small number of coefficients, i.e. most channel coefficients are nearly zero and do not contribute significantly, and the number of effective channel coefficients is relatively much lower than the channel delay spread (length of the channel impulse response). The sparseness of the channel leverages the application of sparse signal processing techniques in the framework of compressive sensing (CS) [5] for channel estimation problems, which can drastically reduce the number of pilots required to estimate the channel, thereby increasing bandwidth efficiency. CS recovery algorithms, such as 11 norm minimization, orthogonal matching pursuit and iterative thresholding, have been adopted for sparse channel estimation, which enables efficient reconstruction of the sparse channel with less pilot overhead than in the case of conventional methods [6]. However, there is no general theory on the optimal pilot selection for sparse channel estimation using CS techniques, such as the optimal equally spaced pilots for conventional channel estimation methods.

A few works of literature deal with the design of a pilot pattern for sparse channel estimation. Deterministic pilot selection [7] is proposed for OFDM systems with the number of subcarriers $N$ being a prime. A pilot generated randomly can be updated by sequentially evolving towards a global optimizer with offline channel data, as discussed in [8]. Pilot design for under water acoustic chan- 
nels, based on cluster pilot design and data subcarrier as additional observation for channel estimation, is described in [9]. The tree-based backward pilot generation [10] iteratively removes a subcarrier from $N$ subcarriers of OFDM systems in a backward direction to generate a pilot pattern. Optimum pilot generation by minimizing coherence of the DFT submatrix and cross entropy optimization, to place the pilots at an optimal location, is described in [11], [12]. The first subcarrier is fixed as the first pilot, and the remaining pilot locations are sequentially assigned by minimizing the variance of the multiset formed using the current pilot location set [13]. Statistic serial, parallel and iterative group shrinking was proposed [14] for minimum coherence. Three greedy deterministic pilot search algorithms [15] are stated based on minimizing coherence through a straightforward search.

The remainder of this paper is organized as follows. Section 2 deals with the OFDM system modeling framework and formulates the estimation problem of sparse channel impulse response. In Section 3, optimal pilot design from the Sylow subgroup is analyzed. The proposed deterministic pilot search algorithm is presented in Section 4. The simulation results shown in Section 5 reveal the performance of the proposed algorithm's pilot pattern in sparse channel estimation of wideband OFDM systems and, finally, conclusions are provided in Section 6.

The notations used in this paper are: $\emptyset, \backslash, \mid, \nmid,(.)^{T},(.)^{H}$, $(.)^{-1},\|\|,.\langle\rangle,. \otimes, \oplus,\lfloor\rfloor,.\lceil$.$\rceil , and O($.$) meaning: empty,$ exclusion, divide, does not divide, matrix transpose, conjugate transpose, matrix inverse, norm of a vector, inner product of a vector, modulus, multiplication modulo, addition modulo, floor of a value, ceiling of a value and order, respectively.

\section{Problem Statement}

In this section, we describe the system model for pilotaided sparse channel estimation in an OFDM system with the canonical discrete time channel model. Assume that information is transmitted through an OFDM symbol that consist of $N$ subcarriers and has a cyclic prefix length of $N / 4$. Among $N$ subcarriers, $N_{p}$ subcarriers are used to transmit pilots with locations represented as $\left[p_{1}, p_{2}, \ldots, p_{N_{p}}\right]$, where $1 \leq p_{1}<p_{2} \ldots<p_{N_{p}} \leq N$ and $N_{d}=N-N_{p}$ subcarriers transmit information. The transmitted and received pilot symbols on pilot subcarrier locations are $x=\left[x\left(p_{1}\right), x\left(p_{2}\right), \ldots, x\left(p_{N_{p}}\right)\right]^{T}$ and $y=\left[y\left(p_{1}\right), y\left(p_{2}\right), \ldots, y\left(p_{N_{p}}\right)\right]^{T}$ respectively. Then the frequency domain sparse channel estimation of a pilot-aided OFDM system at the receiver can be modeled as:

$$
y=A h+n,
$$

where $A=X . F_{N_{p} \times L}$ is a sensing matrix. $X$ is a diagonal matrix of transmitted pilot symbols $X=\operatorname{diag}\left\{x\left(p_{1}\right)\right.$, $\left.x\left(p_{2}\right), \ldots, x\left(p_{N_{p}}\right)\right\}$ and $F_{N_{p} \times L}$ is a discrete Fourier submatrix constructed by selecting first $L$ columns and $N_{p}$ rows stated by pilot locations $\left[p_{1}, p_{2}, \ldots, p_{N_{p}}\right]$ from a standard Fourier matrix $N \times N$.

$$
F_{N_{p} \times L}=\frac{1}{\sqrt{N}}\left[\begin{array}{cccccc}
1 & \omega^{p_{1}} & . & . & . & \omega^{p_{1}^{(L-1)}} \\
1 & \omega^{p_{2}} & . & . & . & \omega^{p_{2}^{(L-1)}} \\
\cdot & \cdot & \cdot & & & \cdot \\
\cdot & \cdot & & \cdot & & \cdot \\
\cdot & \cdot & & & \cdot & \cdot \\
1 & \omega^{p_{N_{p}}} & . & . & . & \omega^{p_{N_{p}}^{(L-1)}}
\end{array}\right]
$$

where $\omega=\mathrm{e}^{-\mathrm{j} \frac{2 \pi}{N}}$ and $n=\left[n(1), n(2), \ldots, n\left(N_{p}\right)\right]^{T}$ is the Additive White Gaussian Noise (AWGN) vector with zero mean and variance $\sigma_{n}^{2} . \quad h=[h(1), h(2), \ldots, h(L)]^{T}$ is the discrete channel impulse response vector with $L$ coefficients. The multipath wireless channel gives rise to multiple attenuated and delayed copies of transmitted signal at the receiver, due to the number of scatters in the surrounding environment. We consider a transmission over such a multipath wireless channel with $L$ resolvable paths (coefficients), and each path has a complex path gain $\alpha_{i}$ and a delay spread $\tau_{i}$. The time domain baseband channel model is given by:

$$
h(\tau)=\sum_{i-1}^{L} \alpha_{i} \delta\left(\tau-\tau_{i}\right)
$$

where $\delta($.$) is a Dirac delta function. The equivalent dis-$ crete channel model can be represented as:

$$
h(n)=\sum_{i=1}^{L} \alpha_{i} \delta\left(\left(n-\tau_{i}\right) T_{s}\right)
$$

where $T_{S}$ is the sampling interval which holds a very small value compared to the maximum delay spread for practical wide band wireless channels. In such cases the impulse response $h$ is dominated by relatively few resolvable paths over the maximum paths $L$, and these channels are often termed as sparse channels. Assuming the frequency domain channel impulse response $h$ is having $\|h\|_{0} \leq k \ll L$, then the multipath wireless channel is termed as $k$ sparse channel.

The reconstruction of channel impulse response $h$ at the receiver is essential for coherent detection. The competent pilot aided channel estimation for today's wireless systems involves either linear or nonlinear techniques. Conventional pilot-aided methods typically depend on linear reconstruction techniques with the resulting sensing matrix $A$ of $N_{p}$ rows and $L$ columns, such that $N_{p}>L$. Considering the inherent sparsity of the wireless channel, the number of pilots $N_{p}$ is kept lower than maximum channel coefficients $L$, i.e. $N_{p}<L$, then system will become an underdetermined system, but improves the system data rate. Thus, linear reconstruction methods do not provide an accurate solution. CS-based methods provide a nonlinear way to reconstruct these under determined systems by exploiting the sparsity of the channel. However, the reliable and efficient reconstruction of sparse vector $h$ by using CS recovery 
techniques is based on proper selection of $N_{p}$ rows for the sensing matrix $A$ from the Fourier matrix, i.e. selection of pilot locations in subcarriers.

\section{Optimal Pilot Analysis}

Proper selection of the pilot pattern influences the sparse channel estimation of OFDM systems using CS reconstruction algorithms. Indeed, sparse vector $h$ is guaranteed for nonlinear reconstruction by CS if $A$ satisfies the Restricted Isometry Property (RIP).

A sensing matrix $A$ satisfies the RIP of order $k$ if there exists a constant $\delta$ such that:

$$
(1-\delta)\|h\|_{2}^{2} \leq\|A h\|_{2}^{2} \leq\left(1_{\delta}\right)\|h\|_{2}^{2},
$$

for any $k$ sparse vector. The minimum of all constants $\delta$ satisfying the above condition is called the isometric constant $\delta k$. But there is no algorithm to check the RIP, since it involves combinatorial computation complexity. Other than RIP, the widely used condition that guarantees the reconstruction of the sparse vector is coherence [16].

Coherence $\mu$ of a sensing matrix $A$, is the largest absolute inner product between any two columns $a_{m}$ and $a_{n}$ of $A$ :

$$
\begin{aligned}
\mu(A) & =\max _{1 \leq m<n \leq L}\left|\left\langle a_{m}, a_{n}\right\rangle\right| \\
& =\left.\max _{1 \leq m<n \leq L}\left|\sum_{i=1}^{N_{p}}\right| x\left(p_{i}\right)\right|^{2} \omega^{p_{i}(n-m)} \mid .
\end{aligned}
$$

Let $c \triangleq n-m$ then

$$
\mu(A)=E \max _{1 \leq c \leq L-1}\left|\sum_{i=1}^{N_{p}} \omega^{p_{i} c}\right|
$$

where energy of pilot $E$ is treated as one.

Now $\omega=\mathrm{e}^{-\mathrm{j} \frac{2 \pi}{N}}=\mathrm{e}^{-\mathrm{j} \theta}$, where $\theta=\frac{q \pi}{N}$.

Then coherence will be:

$$
\begin{aligned}
\mu(A) & =\max _{1 \leq c \leq L-1}\left|\sum_{i=1}^{N_{p}} \mathrm{e}^{-\mathrm{j} c p_{i} \theta}\right| \\
& =\max _{1 \leq c \leq L-1}\left(N_{p}+\sum_{i=1}^{N_{p}} \sum_{j=i+1}^{N_{p}} 2 \cos \left(c\left(\left(p_{i}-p_{j}\right) \bmod N\right) \theta\right)^{\frac{1}{2}}\right. \\
\mu(A) & =\max _{1 \leq c \leq L-1}\left(N_{p}+H_{c}(P)\right)^{\frac{1}{2}}
\end{aligned}
$$

where

$$
H_{c}(P)=\sum_{i=1}^{N_{p}} \sum_{j=i+1}^{N_{p}} 2 \cos \left(c\left(\left(p_{i}-p_{j}\right) \bmod N\right) \theta\right) .
$$

A different pilot pattern leads to a different sensing matrix $A$. The objective function $Q$ for selecting an optimal pilot pattern $P$ is to minimize the coherence of $A$ :

$$
Q=\underset{A}{\arg \min } \mu(A)
$$

A pilot pattern yields minimum coherence $\mu(A)$ if $\cos \left(c\left(p_{i}-p_{j}\right) \theta\right)$ holds minimum for all values of $c$ in Eq. (8). If the most of angles $c\left(p_{i}-p_{j}\right) \theta$ are occupied in the 2nd and 3rd quadrant, then the cosine angle holds minimum. However, for some specific settings, the optimal pilot can be generated from the Cyclic Difference Set (CDS).

Definition for CDS: For the given $\left(N, N_{p}\right)$, if $\lambda$ is an integer where $\lambda=\frac{N_{p}\left(N_{p}-1\right)}{N-1}$ and $N_{p}<N$, then CDS is defined as a set of $N_{p}$ distinct components selected from $N$ denoted as $\left(p_{1}, p_{2}, \ldots, p_{N_{p}}\right)$ satisfying that any integer $x(1 \leq x \leq N-1)$ repeats $\lambda$ times in the set:

$$
\left\{x=p_{i}-p_{j}(\bmod N) \mid 1 \leq i \neq j \leq N_{p}\right\},
$$

and the corresponding difference multiset $D=\left\{a_{d}\right\}_{d=1}^{N-1}$, where $a_{d}$ is the number of pairs $\left(p_{i}, p_{j}\right)$ in pilot indices set $P$ such that $d=p_{i}-p_{j}(\bmod N) \mid 1 \leq i \neq j \leq N_{p}, d=$ $1,2, \ldots, N-1$. The mean and variance $\sigma_{P}^{2}$ of the difference multiset are defined as:

$$
\begin{aligned}
& \text { mean }=\frac{1}{N-1} \sum_{i=1}^{N-1} a_{d_{i}} \\
& \sigma_{P}^{2}=\sum_{i=i}^{N-1}\left(a_{d}-\text { mean }\right)^{2}
\end{aligned}
$$

The pilot selection satisfying the definition of CDS is surely the optimal choice for minimum coherence, since the resultant Discrete Fourier Transform (DFT) submatrix achieves the Welch bound. The sufficient condition for CDS to be optimal pilot selection is:

$$
L \geq\left\lceil\frac{N}{2}\right\rceil \text {. }
$$

Nevertheless, for many pairs of $N$ and $N_{p}$, there is no CDS. Moreover the channel impulse response length $L$ is less than the cyclic prefix of the practical OFDM system.

In the present work for some specific settings, the optimal pilot selection from a p-Sylow subgroup is analyzed and conditions are obtained for a subgroup to act as an optimal pilot selection.

Definition for p-Sylow: Let $(G,$.$) be a group and p$ be a prime number. If $(G)=p^{a} m$, where $p \nmid m$, then a subgroup of order $p^{\alpha}$ is called the p-Sylow subgroup of $G$. Existence of the p-Sylow subgroup is guaranteed. If $(G,$. is an abelian group, then the p-Sylow subgroup is unique. Let $S \subseteq G$. The subgroup of $G$ generated by $S$ is the smallest subgroup of $G$ containing $S$ and is denoted by $(S)$. $(S)$ is the set of finite products of elements of $S$ and its inverses. For any subgroup $H$ of an abelian group $(G,$.$) and for any$ $a \in G, a H=\{a . h \mid h \in H\}$ denotes the coset of $H$ in $G$.

Now, for a given $\left(N, N_{p}\right)$, the optimal pilot selection that can be generated from a subgroup is analyzed. If $N$ and $N_{p}$ satisfy the conditions stated in the following theorem, then the p-Sylow subgroup will be an optimal pilot selection. 
Theorem 1: For a given $\left(N, N_{p}\right)$, if $(N+1)$ is a prime number $q$ and for some prime $p$ and $N_{p}=p^{\alpha}$ for some integer $\alpha$ such that $p^{\alpha} \mid N, p^{\alpha+1} \nmid N$ with $N_{p}\left(N_{p}-1\right)=\lambda N$, where $\lambda$ is an integer and if $P$ is the unique p-Sylow subgroup of the group $(G, \otimes)$ under multiplication $\bmod (N+1)$, then $P$ is a CDS and $P$ is the optimal pilot selection for $\left(N, N_{p}\right)$ for $L \geq \frac{N}{2}$ provided given $p_{1}, p_{2}, p_{3} p_{4} \in P$, there exist $p_{1}^{\prime}, p_{2}^{\prime}$ such that:

$$
\left(p_{1}+p_{2}\right)-\left(p_{3}+p_{4}\right) \equiv\left(p_{1}^{\prime}-p_{2}^{\prime}\right) \bmod (N+1),
$$

where $G=\{1,2, \ldots, N\}$.

Proof: Let $F=\{0,1,3, \ldots, N\}$ and $\lambda=1$. Then $(F, \oplus, \otimes)$ is a field where $\oplus$ and $\otimes$ are the addition $(N+1)$ and multiplication $\bmod (N+1)$ respectively. Let $P=\left(p_{1}, p_{2}\right.$, $\left.p_{3}, \ldots, p_{N_{p}}\right)$ be the unique p-Sylow subgroup of $(G, \otimes)$. Let $\left.S \triangleq\left(p_{i}-p_{j}\right) \bmod (N+1), p_{i} \neq p_{j}\right\}$, where $r \in\{1,2$, $\ldots, N\}$. Clearly, $S$ is non empty and $O(S) \leq N_{p}\left(N_{p}-1\right)=$ $N$. Let $(S)$ be the subgroup of $(F, \oplus)$ generated by $S$. Now, we show that $(S)$ is closed under $\otimes$.

Let $x, y \in(S)$. Suppose $x=s_{1}+s_{2}$, where $s_{1}, s_{2} \in S$. Then $x=\left\{\left(p_{1}-p_{2}\right)+\left(p_{3}-p_{4}\right)\right\} \bmod (N+1)$, where $p_{i} \in P$.

Similarly $y=\left\{\left(p_{1}^{\prime}-p_{2}^{\prime}\right)+\left(p_{3}^{\prime}-p_{4}^{\prime}\right)\right\} \bmod (N+1)$, where $p_{i}^{\prime} \in P$.

Using the given condition, we can find $p_{i}^{\prime} p_{j}^{\prime}$ in $P$ such that $x . y=\left(p_{i}^{\prime}-p_{j}^{\prime}\right) \bmod (N+1)$ for some $p_{i}^{\prime}, p_{j}^{\prime} \in P$. Hence $(S)$ is a subring of $F$. Since $F$ has no proper subring, $(S)=F$. Now we show that $(S) \backslash\{0\}=S$. Let $y(\neq 0) \in(S)$ and let $y=x_{1}+x_{2}$ where $x_{1}, x_{2} \in S$.

Let $x_{1}=\left(p_{1}-p_{2}\right) \bmod (N+1)$ and $x_{2}=\left(p_{3}-p_{4}\right) \bmod (N+1)$. Then:

$$
\begin{aligned}
x_{1}+x_{2} & =\left\{\left(p_{1}+p_{2}\right)-\left(p_{3}+p_{4}\right) \bmod (N+1)\right. \\
& =\left(p_{1}^{\prime}-p_{2}^{\prime}\right) \bmod (N+1), \text { for some } p_{1}^{\prime}, p_{2}^{\prime} \in P, \\
& =x_{3}, \text { for some } x_{3} \in S ; \text { thus }(S) \backslash\{0\}=S .
\end{aligned}
$$

Hence, $S=1,2 \ldots N$. Therefore, $P$ is also a CDS and the optimal pilot selection. For any integer $\lambda \neq 1$ the proof is similar.

Corollary: For a given $\left(N, N_{p}\right)$ if $N$ is a prime number $q$ and for some prime $p$ and for some integer $\alpha$ such that $p^{\alpha} \mid q-1, p^{\alpha+1} \nmid q-1$ with $N_{p}\left(N_{p}-1\right)=\lambda(N-1)$ and $P$ is the unique p-Sylow subgroup of $(G, \otimes)$, under multiplication $\bmod (N)$ where $G=1,2, \ldots, N-1$ such that $P$ satisfies the condition given in theorem 1 then $P$ is the optimal solution.

For the case $\left(N, N_{p}\right)$ where $(N+1)$ is a prime, $N_{p}$ pilots will be selected from $1,2,3, \ldots, N$ whereas in the case $\left(N, N_{p}\right)$, where $N$ is a prime, $N_{p}$ pilots will be selected from $1,2,3, \ldots, N-1$.

The optimal pilot selection for an OFDM system from p-Sylow satisfies the definition of CDS for some specific pairs of $\left(N, N_{p}\right)$. It is no longer an optimal pilot selection for a practical OFDM system with channel length $L<\left\lceil\frac{N}{2}\right\rceil$. For example, $\{1,7,9,10,12,16,26,33,34\}$ is a 3 -Sylow subgroup which is a CDS for $(37,9)$. It has the coher- ence $\mu=2.6458$ for all range of $L \geq\left\lceil\frac{N}{2}\right\rceil$. But if $L=$ $11<\left\lceil\frac{N}{2}\right\rceil$ there exist a set which is neither a subgroup nor a $\operatorname{CDS}\{1,4,7,10,13,22,28,31,34\}$ having coherence $\mu=2.1196$ less than obtained by p-Sylow subgroup and CDS. Practical OFDM systems will have a $\frac{N}{4}$ long cyclic prefix which is usually much larger than the length of the channel. Therefore, it is necessary to explore a pilot search algorithm suitable for wideband OFDM systems.

\section{Pilot Search Algorithm}

This section describes the deterministic procedure for selecting the pilot locations for a given $\left(N, N_{p}, L\right)$. Optimal $N_{p}$ subcarrier selection from $N$ subcarriers of an OFDM system by exhaustive search among all possible ${ }^{N} C_{N_{p}}$ DFT submatrices is humanly impossible because of its computational complexity. Here, a deterministic approach is proposed that couples variance and coherence minimization to meet the near-optimal pilot selection. The algorithm starts by assigning the first pilot location and selects the remaining locations, one by one, so that a difference multiset of selected pilot locations achieves minimum variance. The minimum variance pilot pattern will not lead to minimum coherence for small $L$. Therefore, the algorithm updates every candidate of the selected pilot pattern for minimum coherence from all pilot subcarrier candidates. If the candidate pilot subcarriers are considered to range from 1 to $N$, there is a possibility for the pilot search algorithm to choose pilot subcarriers close to each other. It decreases the efficiency of the pilot pattern in channel estimation [15]. By defining the neighboring optimal distance for pilot subcarriers, the algorithm will not choose the closest subcarriers. In the proposed algorithm index set $I_{S}$ defined to contain candidate pilot subcarrier locations at optimal distance. The optimal distance of pilot subcarriers for the given $N$ and $L$ is $c=\left\lfloor\frac{N}{L}\right\rfloor$.

The proposed algorithm is summarized as Algorithm 1. Initially, the first pilot location is assigned as number one. Next, the second pilot location is selected from the subset $\widehat{P}_{y}$ generated using $\widehat{P}_{y-1} \cup n \mid n \in\left\{I_{s}\right\} \backslash P_{y-1}$ for minimum variance. By repeating this, we obtain the pilot pattern $P=\left[p_{1}, p_{2} \ldots, p_{N_{p}}\right]$. The minimum variance calculation for subset $\widehat{P}_{y}=\left\{P_{y}(1), P_{y}(2), \ldots, P_{y}(n)\right\}$ for each update is given by Eqs. (15)-(19).

Let $P_{y}=\left[p_{1}, p_{2}, \ldots, p_{x}\right]$, where $x \leq N_{p}$ and $y=1,2, \ldots, n$. Then:

$$
\text { mean }=\frac{1}{N-1} \sum_{i=1}^{N-1} a_{d_{i}},
$$

where $a_{d}$ is a element of $D=\left\{a_{d}\right\}_{d=1}^{N-1}$ defined as number of pairs $\left(p_{i}, p_{j}\right)$ in selected pilot location set $P_{y}$ such that $d=$ $p_{i}-p_{j}(\bmod N) \mid 1 \leq i \neq j \leq x$, where $d=1,2, \ldots, N-1$, and variance

$$
\delta_{P_{y}}^{2}=\sum_{i=1}^{N-1}\left(a_{d_{i}}-\text { mean }\right)^{2}
$$




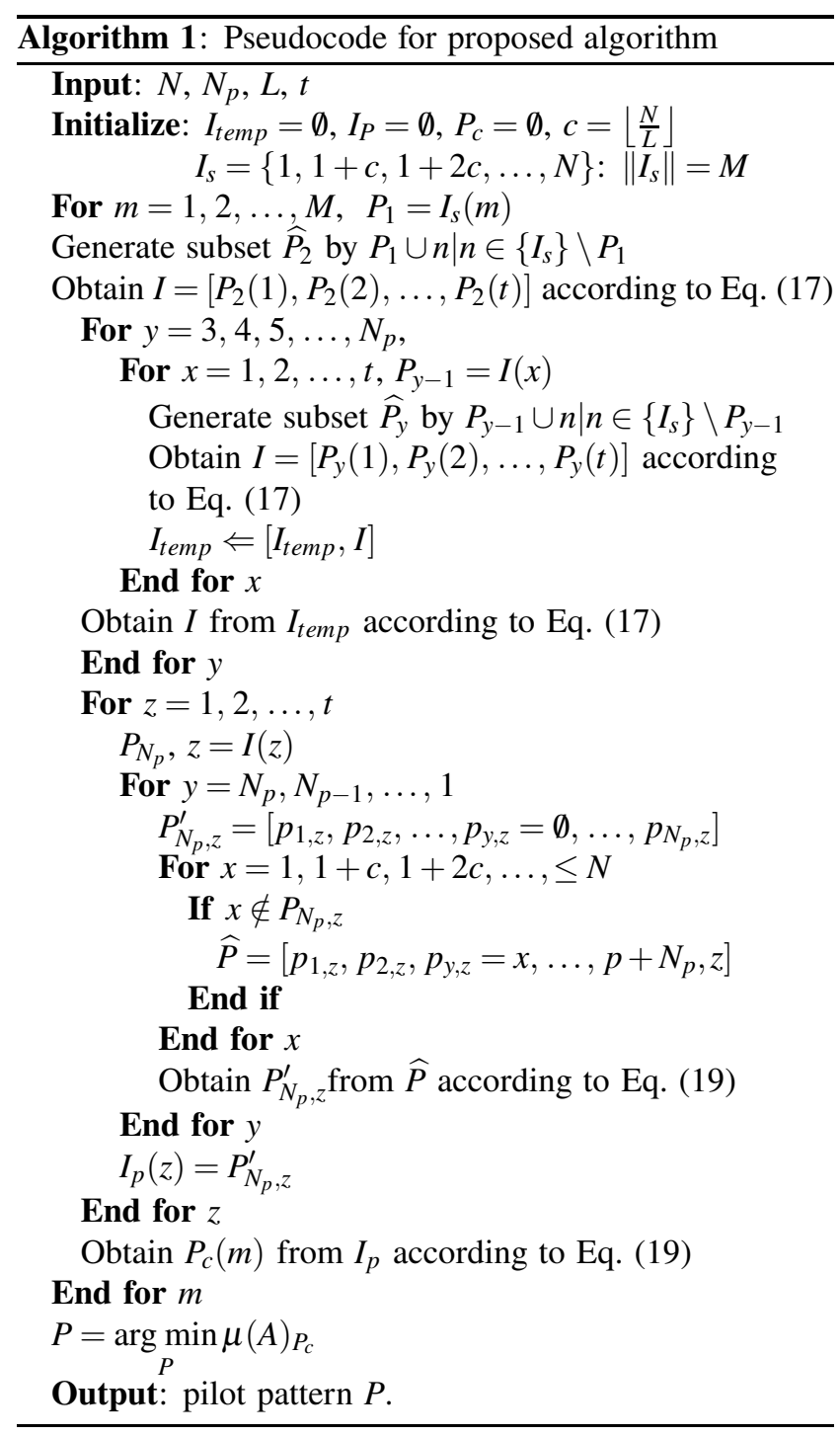

To achieve a greater degree of accuracy, instead of selecting a pilot pattern with the least variance, we select $t$ sets of pilot patterns (group selection) having a minimum variance in every pilot location update. The $t$ sets are selected with least $\sigma_{P_{y}}^{2}$ among $n$ updated set $P=\left[P_{y}(1), P_{y}(2), \ldots, P_{y}(n)\right]$ as:

$$
I=\left[P_{y}(1), P_{y}(2), \ldots, P_{y}(t)\right]
$$

where $y=2,3,4, \ldots, N_{p}$ and $P_{y}(1)=P_{y}(i), P_{y}(2)=$ $P_{y}(j) \ldots \mid 0 \leq \sigma_{P_{y}(i)}^{2} \leq \sigma_{P_{y}(j)}^{2} \leq \ldots$.

The procedure is repeated for every one of the values from index set $I_{S}$ as first pilot location. The resulting $t$ sets of pilot pattern obtained from the first loop is:

$$
I=\left[P_{N_{p}}(1), P_{N_{p}}(2), \ldots, P_{N_{p}}(t)\right]
$$

where $P_{N_{p}}(i)=\left[p_{1, i}, p_{2, i}, \ldots, p_{N_{p}, i}\right]$. All $t$ selected pilot patterns are updated for minimum coherence. Every $n$-th entry of a pilot pattern $P_{N_{p}}$ can be updated by selecting the best form $I_{s} \backslash\left\{P_{N_{p}}(j) \mid j=1,2, \ldots, N_{p}, j \neq n\right\}$. From the resulting pilot location collection set $P$, select a pilot location with minimum coherence as:

$$
P \Leftarrow \underset{P}{\arg \min } \mu(A)_{P(i)}, i=1,2,3, \ldots .
$$

\section{Simulation Results}

The theoretical distance between the pilot locations is verified practically by simulating the proposed algorithm for

Table 1

Coherence of a pilot pattern generated with various distances selected between pilot subcarriers

\begin{tabular}{|c|c|c|c|c|}
\hline$N$ & $N_{p}$ & $L$ & Distance & $\mu$ \\
\hline \hline \multirow{3}{*}{256} & \multirow{3}{*}{16} & & 1 & 4.6868 \\
\cline { 4 - 5 } & & \multirow{3}{*}{50} & 3 & 4.8937 \\
\cline { 4 - 5 } & & & 4 & 4.4308 \\
\cline { 4 - 5 } & & & $\mathbf{5}$ & $\mathbf{4 . 3 8 8 7}$ \\
\cline { 4 - 5 } & & & 6 & 10.8125 \\
\hline
\end{tabular}

Table 2

List of pilot patterns generated for various $\left(N, N_{p}, L\right)$, using the proposed algorithm

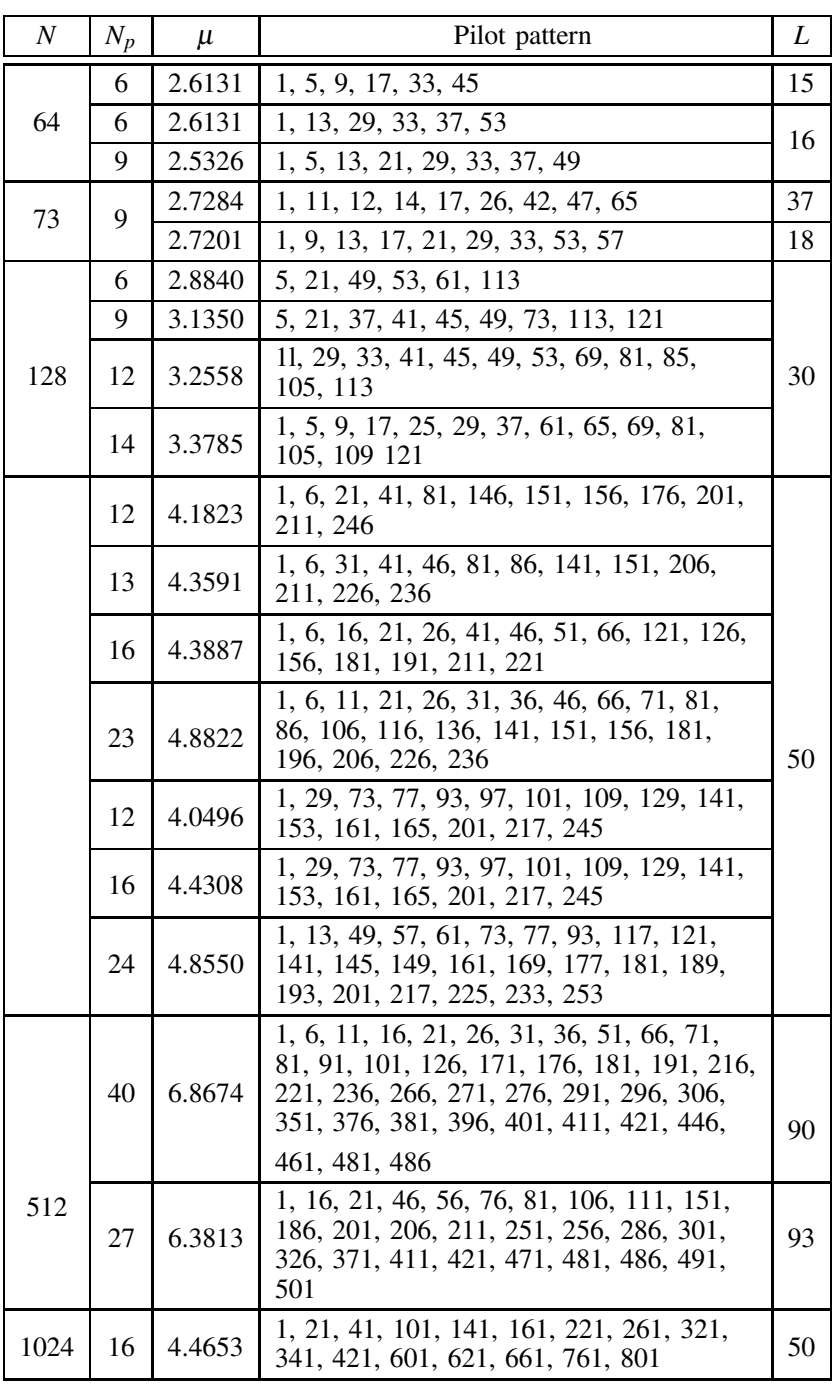


various distances. Table 1 proves that the minimum distance that should be maintained between the pilot locations is $\left\lfloor\frac{N}{L}\right\rfloor$ which is 5 for $N=256$ and $L=50$.

The pilot patterns generated using the proposed algorithm for various pairs of $\left(N, N_{p}, L\right)$ are presented in Table 2. The results show that if $N$ is a prime, coherence of the proposed algorithm's pilot pattern meets the Welch lower bound and prove the effectiveness of the proposed algorithm. For example, $\left(N, N_{p}, L\right)=(73,9,37)$ coherence of the proposed algorithm's pilot location achieves the Welch bound 2.8284. Comparison of coherence obtained by the proposed algorithm with algorithms used in practice for various $\left(N, N_{p}, L\right)$ values is presented in Table 3.

Table 3

Comparison of coherence obtained by the proposed algorithm and by existing pilot search algorithms

\begin{tabular}{|c|c|c|l|c|}
\hline$N$ & $N_{p}$ & $L$ & \multicolumn{1}{|c|}{ Algorithm } & $\mu$ \\
\hline \hline \multirow{3}{*}{256} & \multirow{3}{*}{16} & \multirow{3}{*}{60} & Stochastic serial search [12] & 4.7021 \\
\cline { 4 - 5 } & & & Greedy deterministic [13] & 4.8630 \\
\cline { 4 - 5 } & & Proposed algorithm & $\mathbf{4 . 4 3 0 8}$ \\
\hline \multirow{3}{*}{256} & \multirow{3}{*}{16} & \multirow{3}{*}{50} & Tree based backward with 11 branches [8] & 4.9189 \\
\cline { 4 - 5 } & & & Greedy deterministic [13] & 4.5261 \\
\cline { 4 - 5 } & & Proposed algorithm & $\mathbf{5 . 8 7 5 7}$ \\
\hline \multirow{2}{*}{256} & \multirow{2}{*}{13} & \multirow{2}{*}{50} & Mahdi-Khosravi \& Saeed-Mashhadi [11] & 5.8757 \\
\cline { 4 - 5 } & & Proposed algorithm & $\mathbf{4 . 3 5 9 1}$ \\
\hline
\end{tabular}

An QPSK OFDM system is constructed with 256 subcarriers and 16 of them are used as pilot subcarriers for pilot-aided channel estimation. A discrete sparse channel $h$ is realized with 5 dominant coefficients, randomly placed over the maximum of 50 channel coefficients. A typical discrete channel realization is shown in Fig. 1. The chan-

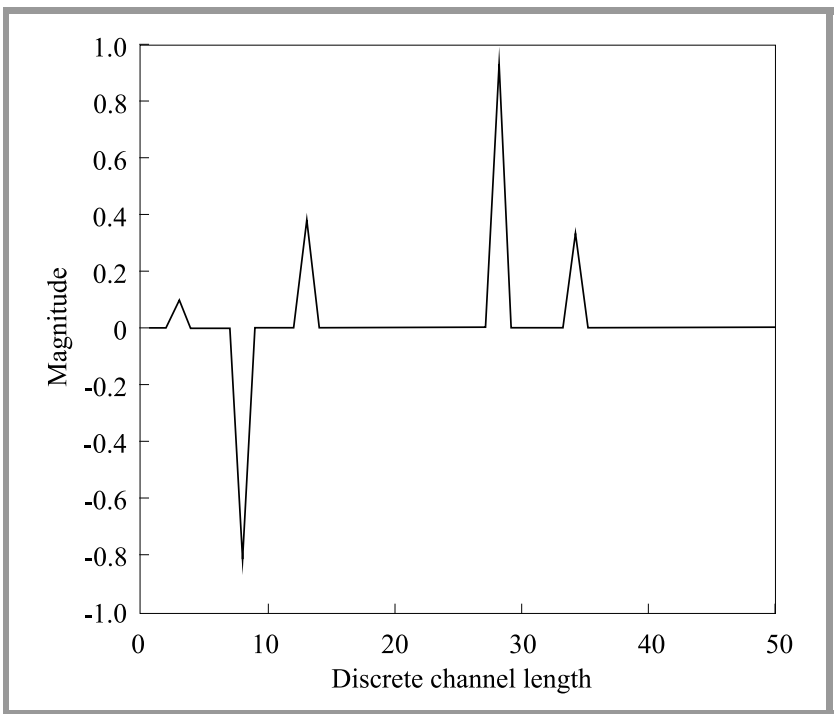

Fig. 1. Typical channel impulse response with 5 dominant coefficients over the maximum discrete channel length. nel estimation performance of the pilot pattern generated using the proposed algorithm is evaluated at the receiver based on the knowledge of the sensing matrix $A$ and the received pilots vector $y$ using greedy iterative orthogonal matching pursuit (OMP) [17]. The normalized mean square error (MSE) associated with channel vector $h$ and estimated channel $\hat{h}$ is calculated by:

$$
M S E=\frac{\|h-\hat{h}\|_{2}^{2}}{\|h\|_{2}^{2}} .
$$

MSE comparison for channel estimation using the proposed and different pilot selection algorithms, comprising, in practice, over 105 iterations for each signal to

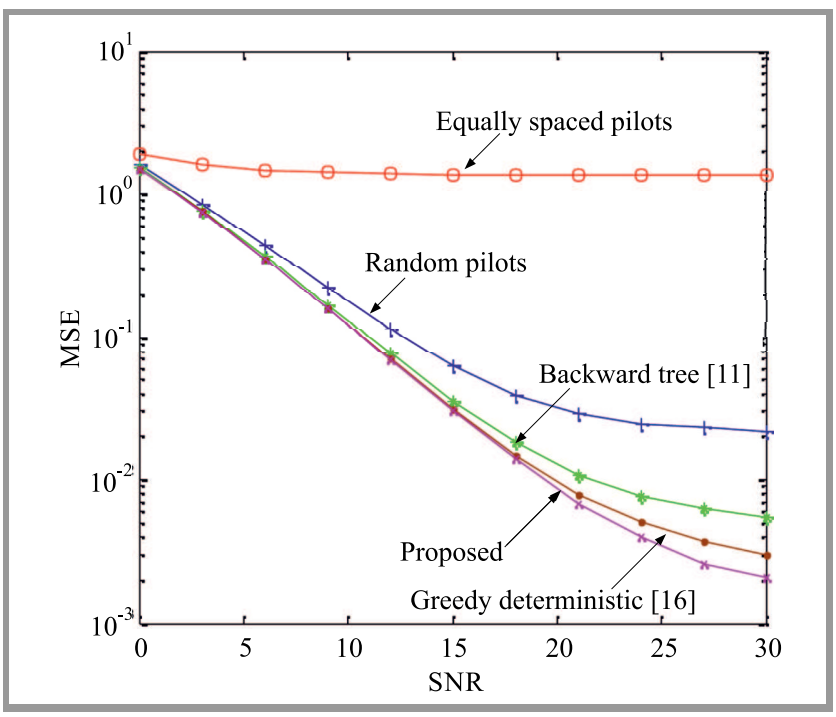

Fig. 2. MSE performance comparison for channel estimation with pilot patterns generated using different schemes for $\left(N, N_{p}, L\right)=$ $(256,16,50)$.

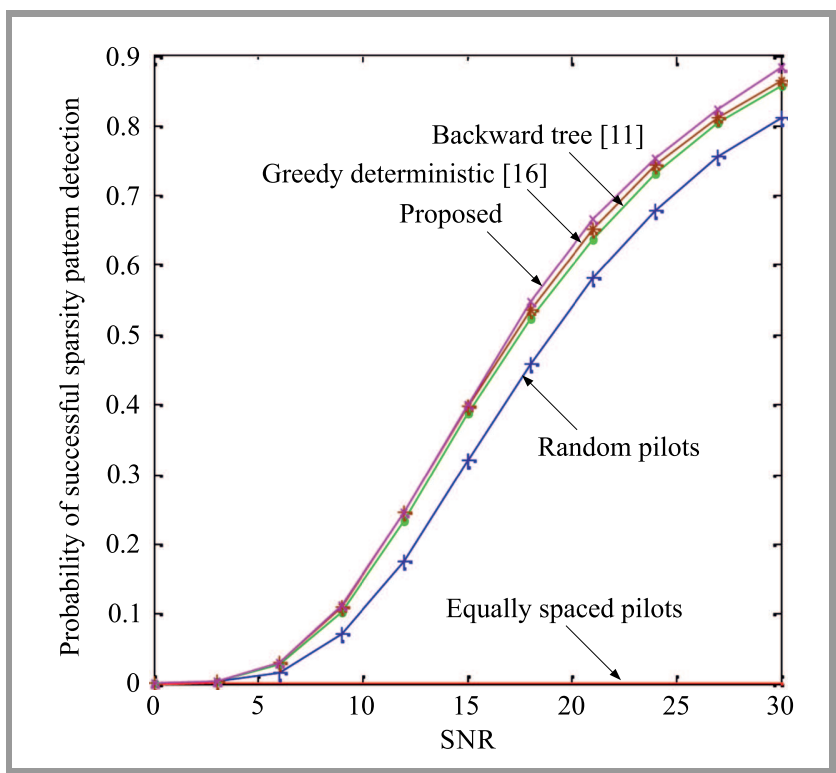

Fig. 3. Detection performance of OMP using proposed and different pilot schemes for $\left(N, N_{p}, L\right)=(256,16,50)$. 
noise ratio (SNR), is plotted in Fig. 2. The equally spaced conventional pilots and random pilots suggested for the sparse channel are also considered for comparison purposes. Equally spaced pilots are not optimized to minimum coherence and there is no benefit in using them for sparse channel estimation and for random pilot generation, which is difficult for practical systems. We observe that the proposed pilot pattern significantly improves the performance of MSE compared to existing schemes used in practice, with reduced coherence. Detection performance of the sparse reconstruction algorithm gives the percentage of cases of exact recovery of the sparse channel from the received pilots. The comparison of sparse channel detection performance of OMP is presented

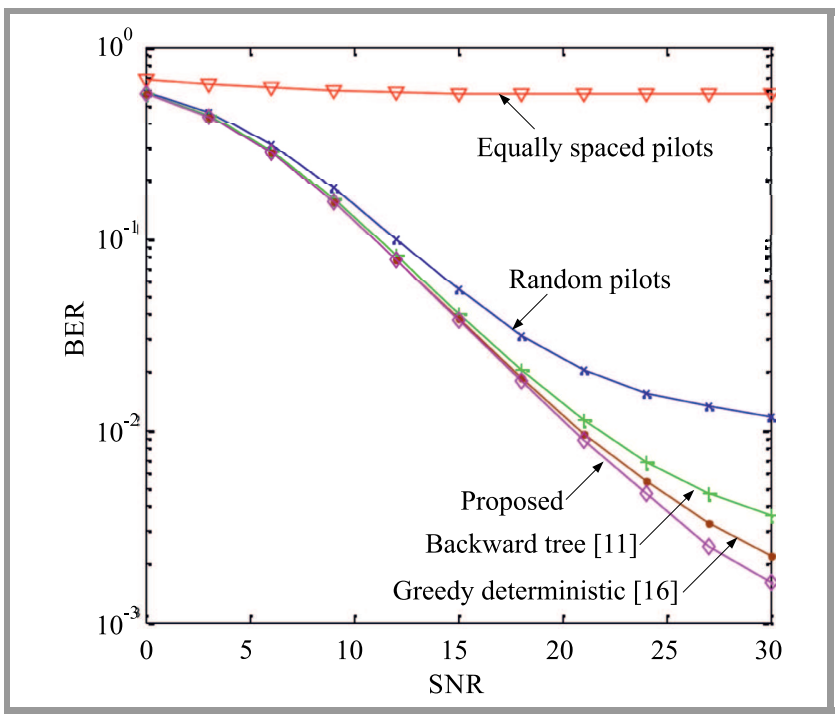

Fig. 4. BER performance comparison of channel estimation for pilot patterns generated using different schemes for $\left(N, N_{p}, L\right)=$ $(256,16,50)$.

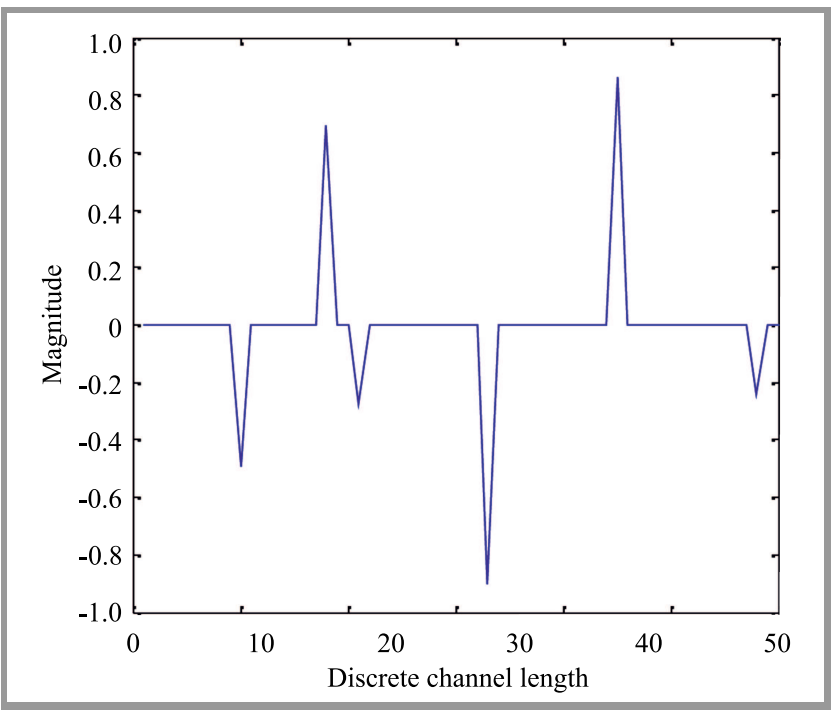

Fig. 5. Typical channel impulse response with 6 dominant coefficients over the maximum discrete channel coefficients $L=60$.

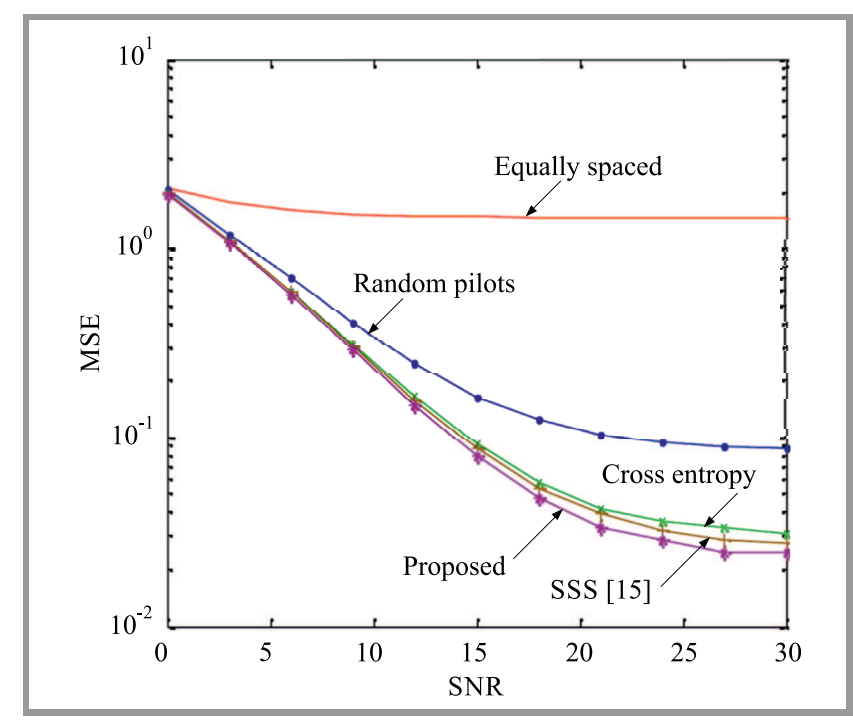

Fig. 6. MSE performance comparison of channel estimation for pilot patterns generated using different schemes for $\left(N, N_{p}, L\right)=$ $(256,16,60)$.

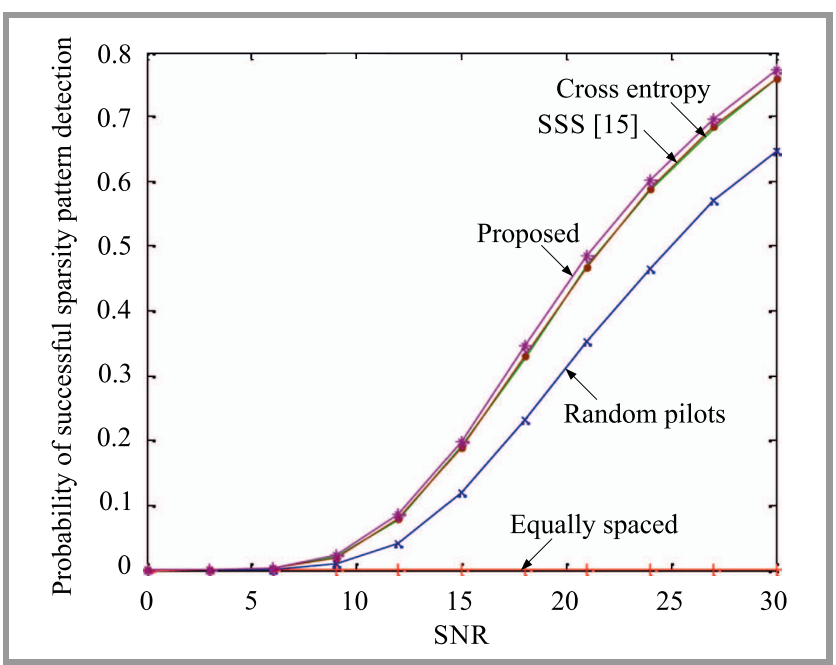

Fig. 7. Detection performance of OMP using proposed and different pilot schemes for $\left(N, N_{p}, L\right)=(256,16,60)$.

in Fig. 3. Transmitted data detection performance is shown in Fig. 4 as Bit Error Rate (BER). Performance of the proposed pilot search algorithm is also evaluated for a channel with 6 dominant coefficients spread over the maximum length of 60 in an QPSK OFDM system channel estimation to verify the robustness of the proposed algorithm. Figure 5 represents a typical channel implementation for $L=60$.

MSE channel estimate, detection performance of the channel estimation algorithm and BER of detected data are given in Figs. 6-8, respectively, for $L=60$ and show the effectiveness of the proposed pilot search algorithm compared to pilot search algorithms used in practice. The running time of the proposed algorithm required to generate pilots with the length of 16 , for the channel length 


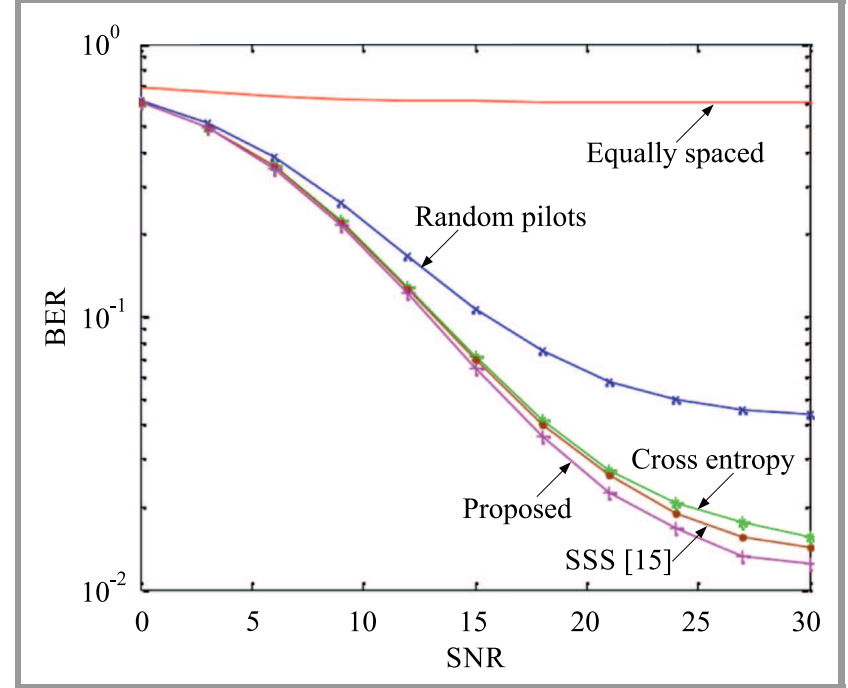

Fig. 8. BER performance comparison of channel estimation for pilot patterns generated using different schemes for $\left(N, N_{p}, L\right)=$ $(256,16,60)$.

of $L=50$ and 60, is 111 and 188 s over 256 subcarriers, respectively.

\section{Conclusions}

In this paper, we investigated pilot selection for pilot-aided sparse channel estimation in wide band OFDM systems. Sufficient conditions are derived to guarantee that p-Sylow is the optimal pilot pattern for some $\left(N, N_{p}, L\right)$. We have proposed a deterministic procedure to select the pilot pattern for given $\left(N, N_{p}, L\right)$. If $N$ is a prime, then the proposed algorithm achieves the Welch bound, confirming its effectiveness. Simulation results show that the pilot pattern generated using the proposed scheme significantly improves the key metrics of wireless systems, including MSE and BER, compared to the existing methods with minimized coherence.

\section{References}

[1] Y. S. Cho, J. K. Won, Y. Y. Chung, and G. Kang, MIMO-OFDM Wireless Communication with MATLAB. Singapore: Wiley \& Sons (Asia), 2010 (doi: 10.1002/9780470825631).

[2] Y. Li, "Pilot-symbol-aided channel estimation for OFDM in wireless systems", IEEE Trans. on Vehicular Technol., vol. 49, no. 4, pp. 1207-1215, 2000 (doi: 10.1109/25.875230).

[3] M. H. Hsieh and Ch. H. Wei, "Channel estimation for OFDM Systems Based on comb-type pilot arrangement in frequency selective fading channels", IEEE Trans. on Consumer Electron., vol. 44, no. 1, pp. 217-225, 1998 (doi: 10.1109/30.663750).

[4] R. Negi and J. Cioffi, "Pilot tone selection for channel estimation in a mobile OFDM system", IEEE Trans. on Consumer Electron., vol. 44, no. 3, pp. 1122-1128, 1998 (doi: 10.1109/30.713244).

[5] D. L. Donoho, "Compressed sensing", IEEE Trans. on Inform. Theory, vol. 52, no. 4, pp. 1289-1306, 2006 (doi: 10.1109/TiT.2006.871582).

[6] W. U. Bajwa, "New information processing theory and methods for exploiting sparsity in wireless Systems", Ph.D. Dissertation, University of Wisconsin, Madison, WI, USA, 2009.
[7] L. Applebaum et al., "Deterministic pilot sequences for sparse channel estimation in OFDM systems", in Proc. of 17th Int. Conf. on Digit. Sig. Process. DSP 2011, Corfu, Greece, 2011, pp. 1-7 (doi: 10.1109/ICDSP.2011.6005021).

[8] Ch. Qi and L. Wu, "Optimized Pilot placement for sparse channel estimation in OFDM systems", IEEE Sig. Process. Lett., vol. 18, no. 12, pp. 749-752, 2011 (doi: 10.1109/LSP.2011.2170834).

[9] Ch. R. Berger, J. Gomes, and J. M. F. Moura, "Study of pilot designs for cyclic-prefix OFDM on time-varying and sparse underwater acoustic channels", in Proc. of IEEE Oceans Conf., Santander, Spain, 2011, pp. 1-8 (doi: 10.1109/Oceans-Spain.2011.6003627).

[10] Ch. Qi and L. Wu, "Tree-based backward pilot generation for sparse channel estimation", Electron. Lett., vol. 48, no. 9, pp. 501-503, 2012 (doi: 10.1049/el.2012.0010).

[11] J. Ch. Chen, Ch. K. Wen, and P. Ting, "An efficient pilot design scheme for sparse channel estimation in OFDM systems", IEEE Commun. Lett., vol. 17, no. 7, pp. 1352-1355, 2013 (doi: 10.1109.LCOMM.2013.051313.122933).

[12] P. Pakrooh, A. Amini, and F. Marvasti, "OFDM pilot allocation for sparse channel estimation", EURASIP J. of Adv. Sig. Process., vol. 59, no. 1, pp. 1-9, 2012 (doi: 10.1186/1687-6180-2012-59).

[13] M. Khosravi and S. Mashhadi, "Joint pilot power pattern design for compressive OFDM channel estimation", IEEE Commun. Lett., vol. 19 , no. 1 , pp. $50-53,2013$

(doi: 10.1109/LCOMM.2014.2371036).

[14] Ch. Qi et al., "Pilot design scheme for sparse channel estimation in OFDM systems", IEEE Trans. on Vehicular Technol., vol. 64, no. 4, pp. 1493-1505, 2015 (doi: 10.1109/TVT.2014.2331085).

[15] S. Hadi, S. Masoumian, and B. M. Tazehkand, "Greedy deterministic pilot pattern algorithms for OFDM sparse channel estimation", Wirel. Personal Commun., vol. 84, no. 2, pp. 1119-1132, 2015 (doi: 10.1007/s11277-015-2679-5).

[16] D. L. Donoho and X. Huo, "Uncertainty principles and ideal atomic decomposition", IEEE Trans. on Inform. Theory, vol. 47, no. 7, pp. 2845-2862, 2001 (doi: 10.1109/18.959265).

[17] A. Ganesh, Z. Zhou, and Y. Ma, "Separation of a subspace signal: Algorithms and conditions", in Proc. of IEEE Int. Con. on Acoust. Speech Sig. Process. ICASSP 2009, Taipei, Taiwan, 2009, pp. 3141-3144 (doi: 10.1109/ICASSP.2009.4960290).

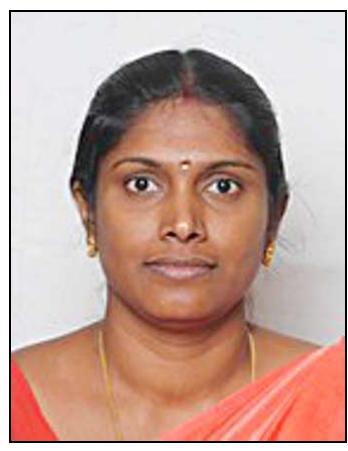

P. Vimala received her B.Sc. in Electronics and Communication Engineering from Jayaram College of Engineering, Trichy, Tamil Nadu, India in 2000 and M.Sc. in Process Control and Instrumentation Engineering from Annamalai University, Chidambaram, Tamilnadu, India in 2010. She is currently working as an Assistant Professor at the Department of Electronics and Communication Engineering, Faculty of Engineering and Technology, Annamalai University. Her current research areas include digital signal processing, sparse signal processing, digital communication and wireless communication.

E-mail: vimalakathirau@gmail.com

Department of Electronics and Communication

Engineering

Annamalai University

Tamil Nadu, India 


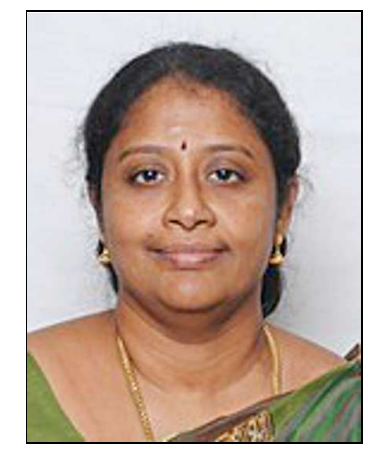

G. Yamuna received her B.Sc. in Electronics and Communication Engineering from National Institute of Technology (NIT), Trichy, Tamil Nadu, India in 1987. She received her M.Sc. in Power Systems from Annamalai University in 1991. She received her Ph.D. in Electrical Engineering from Annamalai University in 2010. She has published many technical papers in national and international conferences and journals. Currently, she is working as a Professor at the Department of Electronics and Communication Engineering, Faculty of Engineering and Technology, Annamalai University, Tamil Nadu, India. She is an associate editor and reviewer for several national and international journals. Her research interests include signal processing, image processing, wireless communication systems, antenna design and information security.

E-mail: yamuna.sky@gmail.com

Department of Electronics and Communication Engineering

Annamalai University

Tamil Nadu, India 